\title{
Ventriculectomia parcial esquerda: ponte para transplante?
}

\author{
José Dario FROTA FILHO*, Fernando A. LUCCHESE*, Celso BLACHER*, Paulo E. LEÃES*, Cídio \\ HALPERIN*, Eraldo A. LÚCIO*, Wagner PEREIRA*, Marcela SALES*, Paul Peter \\ LUNKENHEIMER $^{* *}$, Klaus REDMANN**, Luis E. VARGAS*, Ralf STUERMER*, Roberto LOBO*, \\ Fabio MOREIRA*, Angela P. BUENO*, Luiz A. JUNG*
}

RBCCV 44205-518

\begin{abstract}
Frota Filho J D, Lucchese F A, Blacher C, Leães P E, Halperin C, Lúcio E A, Pereira W, Sales M, Lunkenheimer P P, Redmann K, Vargas L E, Stuermer R, Lobo R, Moreira F, Bueno A P, Jung L A - Ventriculectomia parcial esquerda: ponte para transplante? Rev Bras Cir Cardiovasc 2000; 15 (4): 320-7.
\end{abstract}

RESUMO: Objetivo: Analisar os resultados e a viabilidade da ventriculectomia parcial esquerda (VPE) como ponte para transplante cardíaco (TX).

Delineamento: Estudo de coorte histórica e prospectivo.

Casuística e Métodos: Cinquenta e três pacientes (pts) foram submetidos a VPE em um período de 5 anos. Destes, 7 pts com contra-indicação inicial ao TX, idades variando de 37 a 64 anos, 5 homens e 2 mulheres, com miocardiopatia dilatada, foram subseqüentemente relistados e transplantados. Foram analisados a fração de ejeção (FE), o diâmetro diastólico final do ventrículo esquerdo (DDFVE), a CF da NYHA, o consumo máximo de oxigênio $\left(\mathrm{VO}_{2}\right.$ máx) e os escores de qualidade de vida (QV) antes da VPE, aos 3 e 6 meses, e pré-transplante.

Resultados: Os valores expressos a seguir referem-se, respectivamente, àqueles obtidos antes da VPE, aos 3 e 6 meses e antes do TX. Variação da CF da NYHA: 3,71 $\pm 0,49,2,57 \pm 1,13$ ( $p=0,011), 3,0 \pm 1,29$ e $3,86 \pm 0,38$. Evolução da FE: $25,17 \pm 6,15,35,5 \pm 8,41(p=0,013), 32,33 \pm 7,12$ e $26,17 \pm 3,76$. Variação do DDFVE: $79,16 \pm 10,85,67,66 \pm 9,2,65,83 \pm 9,57$ e $64,25 \pm 8,99$. O VO 2 máx era de $8,12 \pm 3,47$ antes da VPE e de $13,2 \pm 7,75$ aos 6 meses $(p=0,068)$. Variação dos escores de $Q V: 4,29 \pm 1,25,3,0 \pm 1,41$ ( $p=0,050), 3,29 \pm 1,8$ e 4,57 $\pm 1,13$. Foram transplantados $7 / 53$ pts $(13,20 \%)$. A sobrevida, até a data do transplante, variou de 7 a 37 meses $(18,71 \pm 11,78$ meses). O seguimento foi de $100 \%$.

Conclusão: A curto prazo melhoraram a CF da NYHA, a QV, o VO 2 máx, o DDFVE e a FE dos pts. Estes resultados sugerem a possibilidade da indicação da VPE como ponte para TX. Entretanto, a mortalidade elevada no primeiro semestre pós-operatório limita a sua indicação rotineira como ponte para TX. Estudos futuros poderão validar ou não esta possibilidade.

DESCRITORES: Ventrículo cardíaco, cirurgia. Transplante cardíaco. Ventrículo cardíaco, cirurgia, métodos.

\section{INTRODUÇÃO}

$\mathrm{Na}$ última década cresceu consistentemente o número de potenciais candidatos a transplante cardíaco (TX), resultando em grande incremento de receptores em lista de espera. Nos Estados Unidos, de 1.030 em 1988 para 3.698 em 1996, enquanto que o número de doadores atingiu um platô em torno de 2.500 por ano, de 1993 a $1996{ }^{\text {(1). As limitações }}$ dos programas de TX, dificultando-os na maioria das vezes em caráter de urgência, envidam que sejam procuradas alternativas para o TX ${ }^{(2)}$ ou ainda pontes para transplante, incluindo pontes farmacológicas, apesar das limitações (3), dispositivos mecânicos de assistência cardiocirculatória ${ }^{(4,5)}$, cardiomioplastia ${ }^{(6,7)}$, valvuloplastia mitral (8) e ventriculectomia parcial

Trabalho realizado no Hospital São Francisco de Cardiologia e Transplantes da Santa Casa de Porto Alegre. Porto Alegre, RS, Brasil.

Apresentado ao $27^{\circ}$ Congresso Nacional de Cirurgia Cardíaca. Rio de Janeiro, RJ, 23 a 25 de março de 2000.

*Do Hospital São Francisco de Cardiologia e Transplantes da Santa Casa de Porto Alegre.

**Da Westfälische-Wilhelms Universität - University Hospital - Münster - Germany.

Endereço para correspondência: José Dario Frota Filho. Rua Prof. Freitas Cabral 305, apto. 602. Porto Alegre, RS, Brasil. CEP 90.690-130. Tel: (51)

3151584. Fax: (51) 3393937.e-mail: dariofrota@cardiol.br 
Frota Filho J D, Lucchese F A, Blacher C, Leães P E, Halperin C, Lúcio E A, Pereira W, Sales M, Lunkenheimer P P, Redmann K, Vargas L E, Stuermer R, Lobo R, Moreira F, Bueno A P, Jung L A - Ventriculectomia parcial esquerda: ponte para transplante? Rev Bras Cir Cardiovasc 2000; 15(4): 320-7.

esquerda (VPE) ${ }^{(9-12)}$. Por outro lado, em pacientes com miocardiopatia dilatada e insuficiência cardíaca crônica avançada, a instabilidade hemodinâmica aguda implica em mortalidade elevada e necessidade urgente de TX (13). Conseqüentemente, cresce a importância de identificar pacientes com alto risco de morte em lista de espera, para que possam eventualmente ser beneficiados com ponte para transplante ou suas alternativas.

Desde as publicações iniciais sobre VPE (9-11), este procedimento vem sendo empregado em caráter de experimentação clínica em vários países e, em alguns Centros, foi incorporado às estratégias rotineiras para tratamento da insuficiência cardíaca refratária. Em nossa Instituição, a VPE foi indicada nos casos refratários a tratamento clínico, na ausência de doador ou quando havia contra-indicação ao TX, de qualquer natureza. Diversas variáveis foram consideradas para fins de análise e inserção adequada do procedimento entre as opções terapêuticas disponíveis no nosso programa de tratamento da insuficiência cardíaca. Como Centro pioneiro e com experiência acumulada em cinco anos de emprego da técnica, constatamos que alguns pacientes, após VPE primária, foram ainda beneficiados com TX cardíaco e isso nos motivou a analisar este grupo de pacientes.

\section{CASUÍSTICA E MÉTODOS}

\section{Delineamento do Estudo}

Todos os pacientes submetidos a VPE obedeceram critérios preeestabelecidos de inclusão e exclusão e foram seguidos e avaliados no pós-operatório, buscando observar a incidência de uma mesma característica na série ou em grupos de pacientes. As variáveis foram analisadas procurando determinar eventuais relações de causa-efeito entre as mesmas. O estudo ficou delineado como de coorte histórica (observacional, contemporâneo) e prospectivo. Não se determinou previamente o tempo de acompanhamento destes pacientes, sendo o mesmo indeterminado, e os desfechos considerados foram o óbito ou o TX.

\section{Inclusão de Pacientes}

Todos os pacientes com insuficiência cardíaca avançada foram avaliados por um único protocolo de entrada. Conforme os resultados desta avaliação, foram encaminhados a tratamento clínico, TX ou VPE. Para este trabalho foram considerados os 53 pacientes submetidos a VPE e os 7 transplantados posteriormente à VPE. Nenhum dos 7 pacientes foi submetido a VPE com indicação protocolar de ponte para transplante, existindo contra-indica- ção para TX à época da VPE, em todos. Em 5/7 pacientes a contra-indicação ao TX era psicossociocultural e em $2 / 7$ esta se devia a hiperresistência arteriolar pulmonar e hipertensão arterial pulmonar. Todos foram submetidos a VPE primária, isto é, com a intenção de instituir tratamento alternativo definitivo para a insuficiência cardíaca. De acordo com a resposta pós-operatória apresentada e a sobrevida obtida, alguns dos 53 pacientes foram sendo reconsiderados como candidatos a TX e incluídos na lista de espera.

\section{Definições e Conceitos}

Ponte para transplante - O conceito de ponte para transplante adotado neste trabalho se relaciona ao intervalo de tempo entre a VPE e o TX, quando fatores psicossocioculturais inicialmente desfavoráveis ao TX poderiam ser neutralizados ou amenizados. Além disso, o conceito engloba também as possíveis reversões das alterações na circulação pulmonar promovidas pela doença (10), que antes contra-indicavam o TX. Em ambos, entretanto, procuramos identificar um possível efeito direto da VPE que possa ter influenciado o resultado.

Classe Funcional Intermitente - Consideramos como enquadrados na classe funcional IV intermitente da NYHA aqueles pacientes que previamente à VPE, com medicação plena, atingiram esta classe e, momentaneamente, por uma ou mais vezes, encontravam-se em classes menores por influência de medidas terapêuticas adicionais e/ou repouso no leito.

\section{Demografia dos Pacientes}

Do universo de 53 pacientes submetidos a VPE no período de dezembro de 1994 a dezembro de 1999 (5 anos), os 7 que foram posteriormente transplantados eram portadores de miocardiopatia dilatada idiopática, com idades variando de 37 a 64 anos, sendo 5 homens e 2 mulheres. Previamente à VPE, apresentavam fração de ejeção (FE) entre 16 e 35 (média de 25,17 ? 6,15\%; mediana $=25$ ), consumo máximo de oxigênio (VO2 máx) de $8,12 \pm 3,14 \mathrm{ml} /$ $\mathrm{kg} / \mathrm{min}$ e diâmetro diastólico final do VE (DDFVE) de 79,16 ( $10,85 \mathrm{~mm}$. Na classe funcional IV da NYHA estavam 5 pacientes e outros 2 na classe III, estes últimos considerados classe IV intermitente (média de $3,71 \pm 0,49$ e mediana $=4)$. Os escores de Qualidade de Vida estavam entre 4 e 6 , para um máximo de 7 (média de 4,29 $\pm 1,25$ e mediana $=4$ ), de acordo com o protocolo já publicado $(10,11)$. As características pré-operatórias desta coorte de 7 pacientes não diferem significativamente das apresentadas pelos demais pacientes da série de 53 VPE, exceto pelo fato de não haver pacientes chagásicos, enquanto que na série global temos quatro deles (14). 
Frota Filho J D, Lucchese F A, Blacher C, Leães P E, Halperin C, Lúcio E A, Pereira W, Sales M, Lunkenheimer P P, Redmann K, Vargas L E, Stuermer R, Lobo R, Moreira F, Bueno A P, Jung L A - Ventriculectomia parcial esquerda: ponte para transplante? Rev Bras Cir Cardiovasc 2000; 15(4): 320-7.

\section{Técnicas Cirúrgicas}

As ventriculectomias foram realizadas com a técnica originalmente descrita por BATISTA et al. (15), modificada em alguns aspectos em nossa Instituição $(10,11,16)$ e os transplantes através da Técnica Bicava (Wythenshawe) (17). Procedimentos associados foram 5 implantes de biopróteses mitrais, 1 anuloplastia mitral e 1 ressecção de mixoma atrial esquerdo.

\section{Métodos de Avaliação Clínica}

As frações de ejeção e os diâmetros diastólicos finais do VE foram analisados antes das ventriculectomias e, a partir de então, mensalmente, através de ecocardiogramas transtorácicos e/ou transesofágicos. Utilizamos ecocardiógrafo HewlettPackard 5500, com imagem harmônica. Para o cálculo da FE foi empregado o método de Simpson. O $\mathrm{VO}_{2}$ máximo foi determinado pelo protocolo de Naughton, sempre que as condições clínicas permitiam, antes da VPE e aos 30, 90 e 180 dias de pósoperatório. A classe funcional da NYHA e os escores de Qualidade de Vida foram determinados pelo protocolo próprio do Serviço, já amplamente divulgado $(10,11,14)$, sendo os pacientes vinculados ao ambulatório de insuficiência cardíaca e avaliados mensalmente pelo mesmo cardiologista.

\section{Análise Estatística}

Utilizamos os testes não paramétricos de Friedman e Cochran, além do teste de pontos com sinal de Wilcoxon, com o programa SPSS versão 7.0, para analisar as variáveis quantitativas, considerando-se valores de " $p$ " menores que 0,05 como significantes. Das medidas de tendência central utilizamos a média aritmética e a mediana e, dentre as de dispersão, utilizamos o desviopadrão. As variáveis qualitativas são expressas em escala nominal.

\section{RESULTADOS}

Cinco pacientes com contra-indicação inicial ao TX, de ordem psicossociocultural, foram reconsiderados para a lista de espera durante o acompanhamento pós-operatório da VPE. Em 2 outros pacientes, com contra-indicação ao TX devida a hiperresistência arteriolar pulmonar e hipertensão arterial pulmonar, houve retorno destes valores para os limites da normalidade nos primeiros 6 meses pósoperatórios da VPE e foram também relistados. No total, foram $7 / 53$ pacientes transplantados, o que representa um porcentual de $13,20 \%$ da casuística geral. No momento em que surgiram doadores compatíveis, os pacientes encontravam-se em seus do- micílios. Nenhum apresentava quadro agudo de instabilidade hemodinâmica necessitando hospitalização urgente ou uso de suporte cardiocirculatório.

A sobrevida a partir da VPE e até a data do TX foi variável, de 7 a 37 meses, com média de 18,71 $\pm 11,78$ meses (Gráfico 1).

As classes funcionais da NYHA melhoraram no período pós-operatório. Em outras palavras, 71,43 $\%$ dos pacientes estavam em classe IV da NYHA antes da VPE, $28,57 \%$ aos 3 meses pós-operatórios e $57,14 \%$ aos 6 meses. Entretanto, na última avaliação antes do TX, 71,42 \% haviam retornado à classe IV (Gráfico 2). A média variou de 3,71 \pm 0,49 antes da VPE (mediana $=4$ ) para $2.57 \pm 1,13$ aos 3 meses (mediana $=2$ ), para 3,0 $\pm 1,29$ aos 6 meses (mediana $=4$ ) e para $3,86 \pm 0,38$ (mediana = 4) na última avaliação antes do TX. Esta variação, considerados principalmente os períodos de 3 e 6 meses, é estatisticamente significante pelo teste de Friedman $(p=0,011)$. Dicotomizando as classes funcionais em 2 grupos, o primeiro incluindo as classes I e II e, o segundo as classes III e IV e, comparando o número de pacientes em cada grupo nos 4 períodos estudados, encontramos melhora importante na classe funcional aos 3 e 6 meses, com $p=0,017$ (teste de Cochran).

A fração de ejeção, antes com média de 25,17 ? $6,15 \%$ (mediana $=25$ ), aos 3 meses era de 35,5 $(8,41 \%$ (mediana $=35)$, aos 6 meses de 32,33 $(7,12 \%$ (mediana $=30)$ e alcançou o valor médio de 26,17 $\pm 3,76 \%$ (mediana $=27$ ) antes do TX (Gráfico 3). A variação aos 3 e 6 meses foi estatisticamente significante, com $\mathrm{p}=0,013$.

O DDFVE pré-VPE era de 79,16 $\pm 10,85$ $\mathrm{mm}$, diminuiu para $67,66 \pm 9,2 \mathrm{~mm}$ aos 3 meses, $65,83 \pm 9,57 \mathrm{~mm}$ aos 6 meses e para $64,25 \pm$ $8,99 \mathrm{~mm}$ imediatamente antes do TX, com $\mathrm{p}=0,04$ (Gráfico 4).

A Qualidade de Vida apresentou variação semelhante à classe funcional da NYHA. Antes da VPE, $6(85,71 \%)$ pacientes receberam escores de 4 a 6 . Aos 3 meses, $42,85 \%$ ou 3 pacientes, estavam com escores entre 4 e 5 e aos 6 meses, 3 $(57,14 \%)$ pacientes voltaram a escores entre 4 e 6 . As médias variaram de $4,29 \pm 1,25$ antes da VPE (mediana $=4$ ), 3,00 $\pm 1,41$ aos 3 meses (mediana $=3$ ), $3,29 \pm 1,80$ aos 6 meses (mediana $=4$ ) e 4,57 $\pm 1,13$ antes do TX (mediana $=4$ ), alcançando valor muito próximo da significância estatística aos 3 e 6 meses ( $p=0,050)$ - (Gráfico 5).

O consumo máximo de oxigênio, antes de 8,12 $\pm 3,47 \mathrm{ml} / \mathrm{kg} / \mathrm{min}$, elevou-se para $13,2 \pm 7,75 \mathrm{ml} / \mathrm{kg} /$ min aos 6 meses pós-VPE, com $p=0,068$ pelo teste de Wilcoxon. Possivelmente, o número pequeno de pacientes em condições clínicas de realizar o exame, em ambos os momentos, tenha interferido no resultado estatístico (Gráfico 6). 
Frota Filho J D, Lucchese F A, Blacher C, Leães P E, Halperin C, Lúcio E A, Pereira W, Sales M, Lunkenheimer P P, Redmann K, Vargas L E, Stuermer R, Lobo R, Moreira F, Bueno A P, Jung L A - Ventriculectomia parcial esquerda: ponte para transplante? Rev Bras Cir Cardiovasc 2000; 15(4): 320-7.

\section{GRÁFICO 1}

TEMPO DE SOBREVIDA, EM MESES, APÓS VPE E ATÉ O TX $(n=7)$

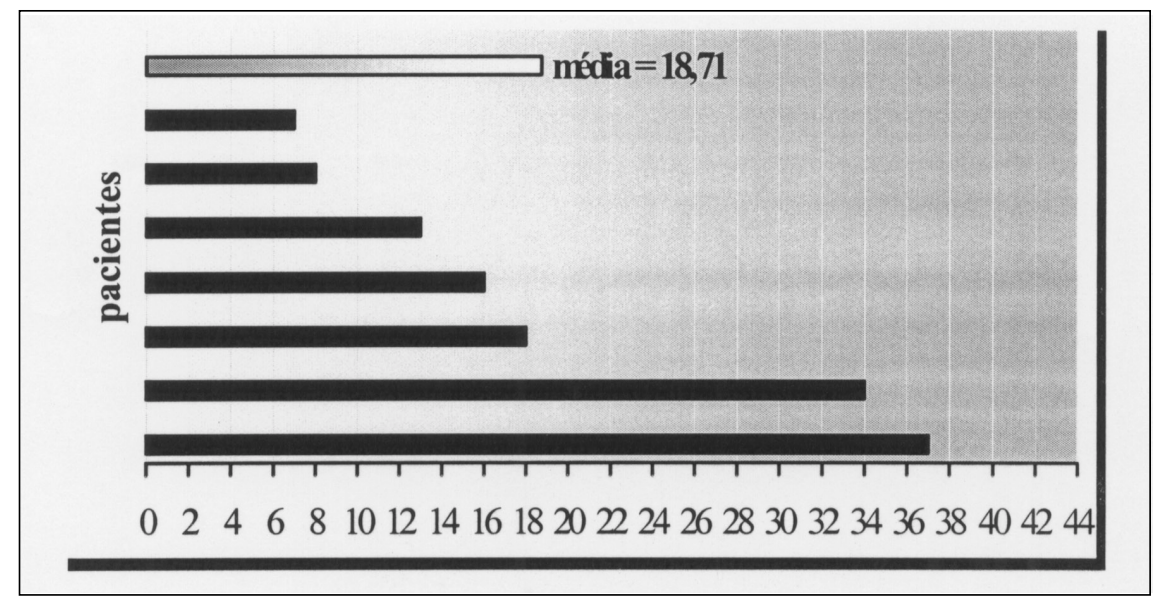

GRÁFICO 2

CLASSES FUNCIONAIS DA NYHA

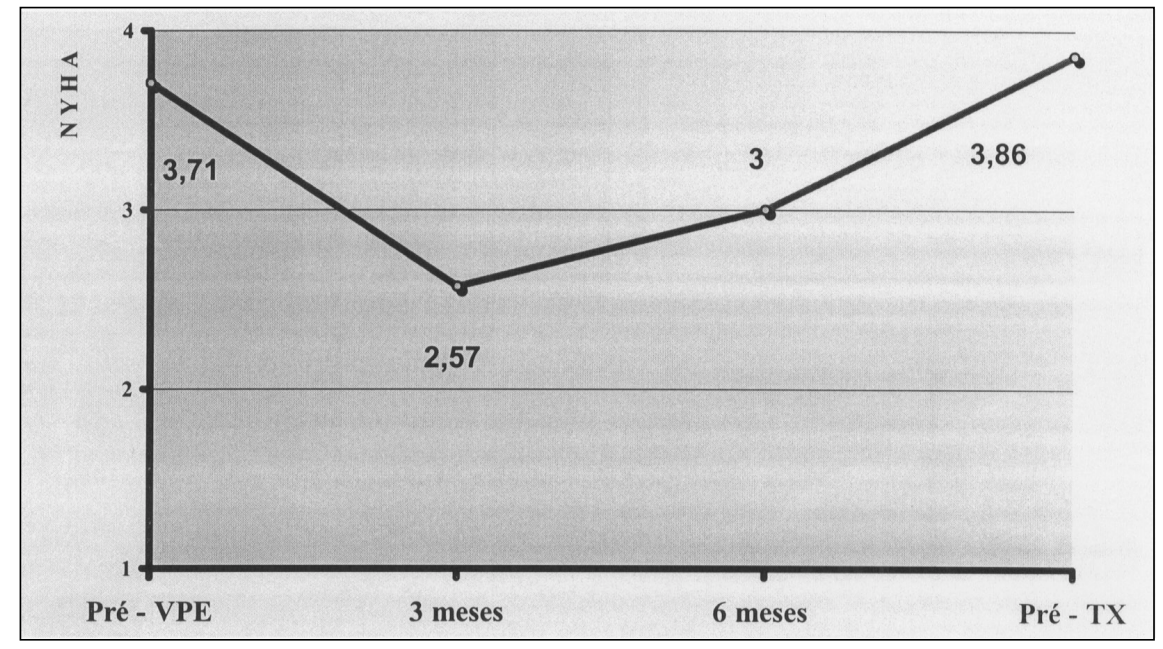

Ao compararmos as características pré e pósoperatórias destes 7 pacientes com os demais pacientes submetidos a VPE, no mesmo intervalo de tempo, não constatamos diferenças significativas no que se refere aos parâmetros aqui analisados, exceto pelo fato de na presente coorte não haver pacientes portadores de doença de Chagas, enquanto que na série geral temos 4 deles (14). A probabilidade de sobrevida, determinada pela curva atuarial de KaplanMeier, é de 31,33 $\pm 6,46$ em 1 ano, 29,24 $\pm 6,36$ em 2 e 3 anos e de 23,39 $\pm 7,3$ aos 4 e 5 anos. Aos 3 e 6 meses pós-operatórios esta probabilidade é de 54,72 $\pm 6,24$ e 47,14 $\pm 6,86$, respectivamente. A maioria dos óbitos ocorreu nos primeiros 6 meses de pós-operatório, com marcada influência dos óbitos imediatos (hospitalares ou 30 dias após a VPE).

\section{COMENTÁRIOS}

Em revisão recente da literatura, relacionada a ponte para transplante cardíaco, constatamos que, mesmo não encontrando uma definição acadêmica e consensual do termo e da condição chamada ponte para transplante, na maioria das vezes esta se refere ao emprego de métodos de suporte cardiocirculatório, na eventualidade de deterioração hemodinâmica aguda, com risco de vida e impossibilidade provisória de TX. Aplica-se, majoritariamente, aos estados de choque cardiogênico pós-infarto agudo do miocárdio, pós-cardiotomia, e instabilidade hemodinâmica de difícil manejo em pacientes com insuficiência cardíaca em lista de espera para TX. Embora todos os pacientes analisados neste trabaIho tivessem contra-indicação ao TX na época da 
Frota Filho J D, Lucchese F A, Blacher C, Leães P E, Halperin C, Lúcio E A, Pereira W, Sales M, Lunkenheimer P P, Redmann K, Vargas LE, Stuermer R, Lobo R, Moreira F, Bueno A P, Jung L A - Ventriculectomia parcial esquerda: ponte para transplante? Rev Bras Cir Cardiovasc 2000; 15(4): 320-7.

\section{GRÁFICO 3 \\ EVOLUÇÃO DA FRAÇÃO DE EJEÇÃO NOS QUATRO PERÍODOS}

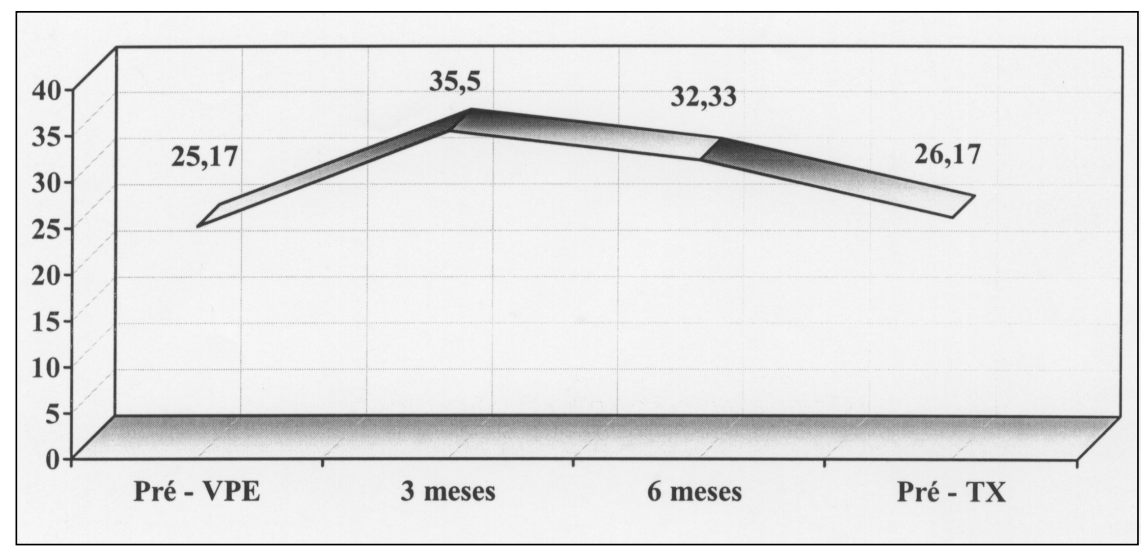

\section{GRÁFICO 4}

EVOLUÇÃO DOS DIÂMETROS DIASTÓLICOS (EM MILÍMETROS)

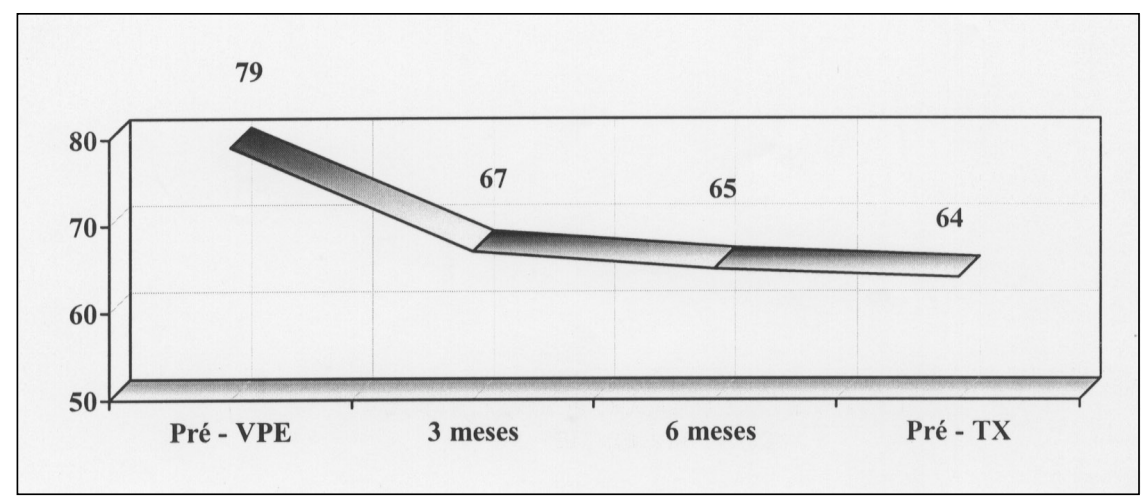

indicação da VPE, nenhum deles apresentava qualquer das situações clínicas acima descritas, revelando-se aqui uma possível limitação do estudo. Entretanto, no intervalo de tempo entre a VPE e o TX, ficou evidenciada a possibilidade da neutralização de fatores psicossocioculturais inicialmente desfavoráveis ao TX e a reversão das alterações na circulação pulmonar promovidas pela doença em 2 pacientes (10), que antes contra-indicavam o TX. Sugerimos que estes benefícios, possíveis de serem alcançados, sejam considerados na definição de ponte para transplante. Consequentemente, torna-se imperativo, doravante, que a avaliação dos meios e métodos utilizados como ponte para transplante considerem não só as condições clínicas momentâneas, mas também os possíveis benefícios que possam advir com procedimentos ditos ponte para transplante.

Os fatores prognósticos para mortalidade elevada em 2 anos, com tratamento clínico otimizado, incluem o $\mathrm{VO}_{2}$ máximo menor que 10 $\mathrm{ml} / \mathrm{kg} / \mathrm{min}$ (1), o DDFVE igual ou maior que 80 $\mathrm{mm}$ e o sódio sérico menor que $132 \mathrm{mEq} / \mathrm{l}$. Estes 2 últimos, podem ser responsáveis por taxas de mortalidade de $71 \%$ e $65 \%$, respectivamente, em 2 anos de lista de espera(18). Os pacientes aqui estudados apresentavam $\mathrm{VO}_{2}$ máximo de 8,12 ? 3,47 $\mathrm{ml} / \mathrm{kg} / \mathrm{min}$ antes da VPE, sendo, portanto, considerados de risco aumentado para mortalidade. Mantiveram valores de 13,2 \pm 7,75 $\mathrm{ml} / \mathrm{kg} / \mathrm{min}$ aos 6 meses após a VPE, o que pôde, provavelmente, sustentar uma condição de risco diminuído. A média dos DDFVE antes da VPE, de $79,16 \pm 10,85 \mathrm{~mm}$, os colocava também em situação de risco elevado. A diminuição para $67,66 \pm 9,2 \mathrm{~mm}$ aos 3 meses, 65,83 $\pm 9,57 \mathrm{~mm}$ aos 6 meses e 64,25 $\pm 108,99 \mathrm{~mm}$ imediatamente antes do TX, sustentada, portanto, pode ter também favorecido a espera por um TX. Redução semelhante nas dimensões do VE ocorreram em praticamente todos as casuísticas até 
Frota Filho J D, Lucchese F A, Blacher C, Leães P E, Halperin C, Lúcio E A, Pereira W, Sales M, Lunkenheimer P P, Redmann K, Vargas L E, Stuermer R, Lobo R, Moreira F, Bueno A P, Jung L A - Ventriculectomia parcial esquerda: ponte para transplante? Rev Bras Cir Cardiovasc 2000; 15(4): 320-7.

\section{GRÁFICO 5}

QUALIDADE DE VIDA

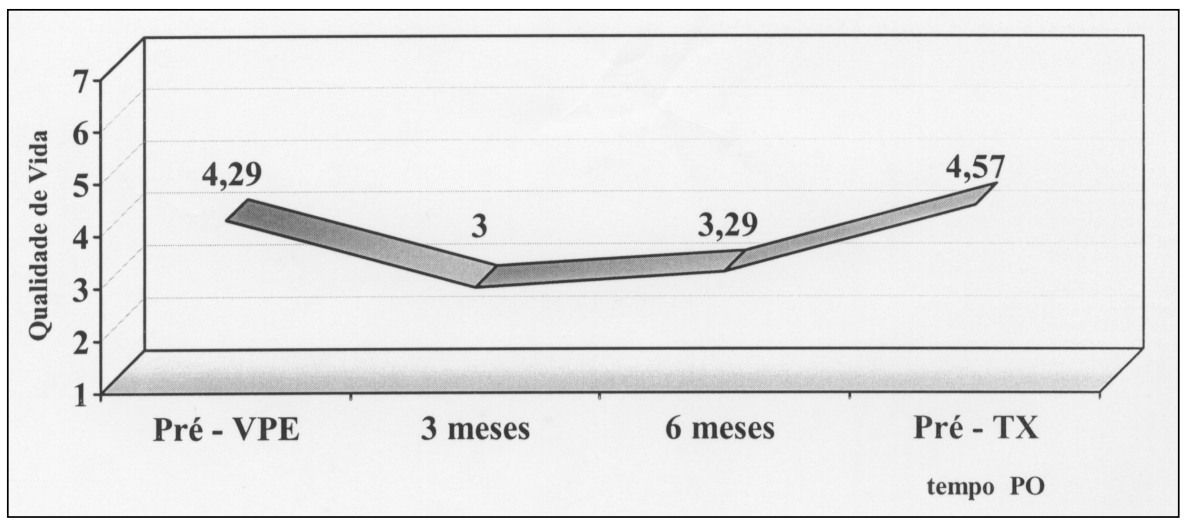

GRÁFICO 6

CONSUMO MÁXIMO DE OXIGÊNIO (ml/kg/min)

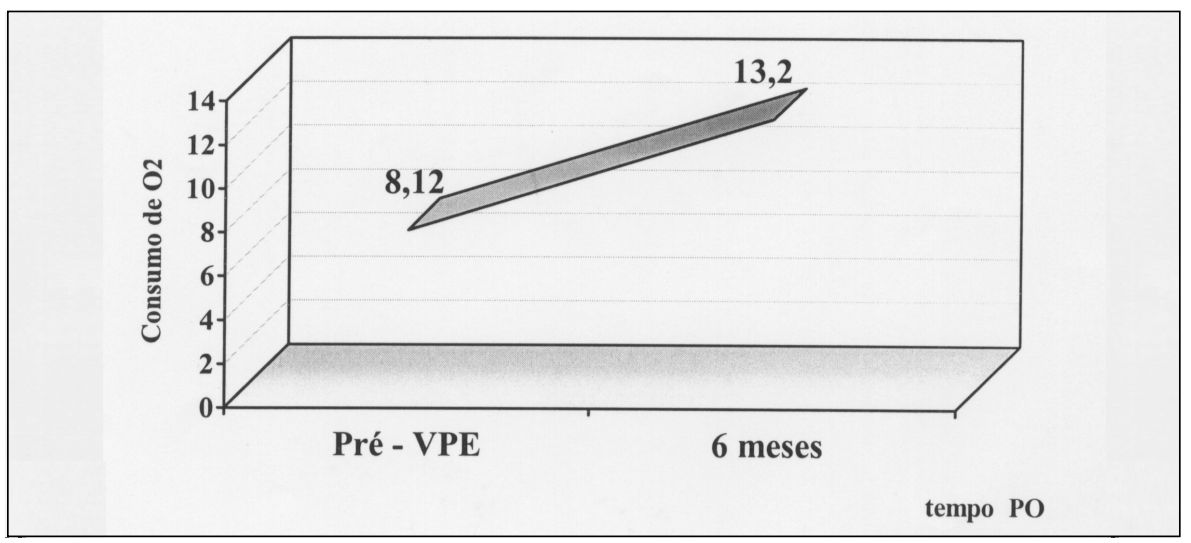

então publicadas ${ }^{(19)}$. Estes achados, à primeira vista, sugerem que a VPE, ao controlar a curto prazo dois dos fatores responsáveis por mortalidade elevada nas listas de espera, possa ser considerada uma opção como ponte para transplante.

A mortalidade imediata e tardia na maioria das séries publicadas de ventriculectomias é elevada $(14,20)$, sendo aproximadamente de $20 \%$ em 30 dias e de $40 \%$ tardiamente, com mortalidade global de $60 \%$ ao final de um ano. Nossos números indicam $54,72 \pm 6,24 \%$ aos 3 meses de pósoperatório e 47,14 $\pm 6,86 \%$ aos 6 meses. Sem dúvida que estas taxas de mortalidade podem levar ao questionamento da validade da indicação rotineira da VPE como ponte para transplante. Este fato nos motivou a reconsiderar todos os pacientes submetidos a VPE, que preencheram adequadamente os critérios, como potenciais candidatos a TX, após rigoroso reestudo.
$\mathrm{Na}$ nossa coorte, o percentual de pacientes transplantados após a VPE foi de 13,20 \% (7/53), o que consideramos ainda muito baixo. A explicação pode estar, de forma parcial, assentada no fato da VPE não ter sido protocolada inicialmente como ponte para transplante, mas sim como tratamento definitivo para os casos com contra-indicação. A melhora clínica e a sobrevida até então alcançada, associada ao retorno dos sintomas (CF IV) e a interferência dos profissionais da equipe multidisciplinar de TX, condicionando os pacientes, foram os fatores responsáveis pela relistagem dos mesmos. Por outro lado, este baixo percentual reflete, principalmente, as muitas dificuldades inerentes aos programas de TX como, por exemplo, falta de doadores. Conseqüentemente, com o aumento do número de receptores e a identificação de fatores de risco para mortalidade elevada nas listas de espera, torna-se necessária a disponibilização de procedimentos que funcionem como ponte para transplante. 
Frota Filho J D, Lucchese F A, Blacher C, Leães P E, Halperin C, Lúcio E A, Pereira W, Sales M, Lunkenheimer P P, Redmann K, Vargas L E, Stuermer R, Lobo R, Moreira F, Bueno A P, Jung L A - Ventriculectomia parcial esquerda: ponte para transplante? Rev Bras Cir Cardiovasc 2000; 15(4): 320-7.

\section{CONCLUSÃO}

Em resumo, os números apresentados evidenciam, a curto prazo, melhora na CF da NYHA, na Qualidade de Vida, no consumo máximo de oxigênio, no DDFVE e na fração de ejeção após a VPE, estando pelo menos duas destas variáveis implicadas em maior mortalidade nas listas de espera. Pacientes com contra-indicação ao TX puderam ser relistados e transplantados. Estes resultados sugerem a possibilidade da indicação da VPE como ponte para transplante. Entretanto, a mortalidade elevada no primeiro semestre pós-operatório e outras questões não esclarecidas (21) limitam, no momento, a sua indicação rotineira como ponte para transplante. Estudos futuros poderão validar ou não esta possibilidade.

RBCCV 44205-518

Frota Filho J D, Lucchese F A, Blacher C, Leães P E, Halperin C, Lúcio E A, Pereira W, Sales M, Lunkenheimer P P, Redmann K, Vargas L E, Stuermer R, Lobo R, Moreira F, Bueno A P, Jung L A - Partial left ventriculectomy: bridge to transplantation? Rev Bras Cir Cardiovasc 2000; 15(4): 320-7.

ABSTRACT:Objective:To assess the suitability of Partial Left Ventriculectomy (PLV) as abridge to transplantation. Background: Cohort study, prospective.

Material and Methods: Fifty-three patients were submitted to PLV in a time frame of 5 years. Seven out of 53 patients, ages ranging from 37 to 64 years old, 5 males and 2 females, all with idiopathic dilated cardiomyopathy, were transplanted afterwards. Ejection fraction, NYHA functional classes, maximum oxygen consumption, left ventricular end-diastolic diameter and Quality of Life Scores were analysed preoperatively and then at three and six months and immediately before transplantation.

Results: The numerical values mentioned below are referred to the data obtained pre-ventriculectomy, at 3 and 6 postoperative months and immediately before transplantation. NYHA functional classes: $3.71 \pm 0.49,2.57$ $\pm 1.13(p=0.011), 3.0 \pm 1.29$ and $3.86 \pm 0.38$. Ejection fraction: $25.17 \pm 6.15,35.5 \pm 8.41(p=0.013), 32.33 \pm 7.12$ and 26.17 \pm 3.76 . Left ventricular end-diastolic diameter: $79.16 \pm 10.85,67.66 \pm 9.2,65.83 \pm 9.57$ e $64.25 \pm 8.99$. Maximum $\mathrm{VO}_{2}$ was $8.12 \pm 3.47$ pre-VPE and increased to $13.2 \pm 7.75$ at six months $(p=0.068)$. Quality of life scores: $4.29 \pm 1.25,3.0 \pm 1.41$ ( $p=0.050), 3.29 \pm 1.8$ e $4.57 \pm 1.13 .7 / 53$ patients $(13.20 \%)$ were subsequently transplanted. Survival time, from PLV up to TX ranged from 7 to 33 months $(18.71 \pm 11.78)$. Follow-up was $100 \%$.

Conclusion: Improvement in quality of life, NYHA functional classes and maximum $\mathrm{VO}_{2}$ consumption, as well as increase in ejection fraction and sustained decrease in left ventricular end-diastolic diameter, in shortterm, can benefit patients previously excluded from TX and bring them back to the awaiting list. However, high immediate mortality rates in overall casuistry can limit its routine indication as a biological bridge to transplantation.

DESCRIPTORS: Heart ventricle, surgery. Heart transplantation. Heart ventricle, surgery, methods.

AGRADECIMENTOS: A Vânia Naomi Hirakata, pela análise estatística, e a Patrícia Muniz, pelo zelo na guarda dos dados do programa de VPE. A ambas, ainda, os maiores cumprimentos pela qualidade do relacionamento profissional.

\section{REFERÊNCIAS BIBLIOGRÁFICAS}

1 Aaronson K D \& Mancini D M - Mortality remains high for outpatient transplant candidates with prolonged (>6 months) waiting list time. J Am Coll Cardiol 1999; 33: 1189-95.

2 Starling R C - Radical alternatives to transplantation. Curr Opin Cardiol 1997; 12: 166-71.

3 Loisance D Y, Deleuze $\mathrm{P} \mathrm{H}$, Houel R et al. Pharmacological bridge to cardiac transplantation: current limitations. Ann Thorac Surg 1993; 55: 310-3.

4 Levin H R \& Weisfeldt M L - Deep thoughts on tin men:

fact, fallacy, and future of mechanical circulatory support. Circulation 1997; 95: 2340-3.

5
Mussivand T, Hendry P J, Masters R G, Kean W J Development of a ventricular assist device for out-ofhospital use. J Heart Lung Transplant 1999; 18: 166-71.

Magovern G J \& Simpson K A - Clinical cardiomyoplasty: review of the ten-year United States experience. Ann Thorac Surg 1996; 61: 413-9.

7 Braile D M, Godoy M F, Thevenard G H et al. - Clinical evolving analysis of patients with dilated cardiomyopathy undergoing dynamic cardiomyoplasty. Progr Biomed Res 1996; 58-64.

Bolling S F, Deeb G M, Brunsting L A, Bach D S - Early outcome of mitral valve reconstruction in patients with end-stage cardiomyopathy. J Thorac Cardiovasc Surg, 1995; 109: 676-83.

Batista R J, Santos J L, Takeshita N, Bocchino L, Lima P N, Cunha M A - Partial left ventriculectomy to improve left ventricular function in end-stage heart disease. J Card Surg 1996; 11: 96-8. 
Frota Filho J D, Lucchese F A, Blacher C, Leães P E, Halperin C, Lúcio E A, Pereira W, Sales M, Lunkenheimer P P, Redmann K, Vargas L E, Stuermer R, Lobo R, Moreira F, Bueno A P, Jung L A - Ventriculectomia parcial esquerda: ponte para transplante? Rev Bras Cir Cardiovasc 2000; 15(4): 320-7.

10 Lucchese F A, Frota Filho J D, Blacher C et al. Ventriculectomia parcial esquerda como ponte para transplante em pacientes com insuficiência cardíaca refratária e hipertensäo pulmonar. Rev Bras Cir Cardiovasc 1997; 12: 221-5.

11 Frota Filho J D, Pereira W M, Leães P E et al. - Endstage heart failure: is there a role for the Batista procedure? Heart Surg Forum 1996; 1: 41-8.

12 McCarthy P M, Starling R C, Wong J et al. - Early results with partial left ventriculectomy. J Thorac Cardiovasc Surg 1997; 114: 755-65.

13 Stevenson L W, Hamilton M A, Tillisch I H et al. Decreasing survival benefit from cardiac transplantation for outpatients as the waiting list lengthens. J Am Coll Cardiol 1991; 18: 919-25.

14 Frota Filho J D, Lucchese F A, Blacher C et al. - Três anos de ventriculectomia parcial esquerda: resultados globais e tardios em 41 pacientes. Rev Bras Cir Cardiovasc 1999; 14: 75-87.

15 Batista R J V, Santos J L V, Franzoni M et al. Ventriculectomia parcial: um novo conceito no tratamento cirúrgico de cardiopatias em fase final. Rev Bras Cir Cardiovasc 1996; 11: 1-6.

16 Blanche C, Frota Filho J D, Trento A, Lucchese F A Technical considerations for partial left ventriculectorny (Batista operation). J Cardiovasc Surg 1998; 39: 829-32.

17 Gamel A El, Yonan N A, Grant S et al. - Orthotopic cardiac transplantation: a comparison of standard and bicaval Wythenshawe techniques. J Thorac Cardiovac Surg 1995; 109: 721-30.

18 Stevenson L W - Patient selection for mechanical bridging to transplantation. Ann Thorac Surg 1996; 61: 380-7.

19 Frota Filho J D, Blacher C, Lucchese F A et al. Ventriculectomia parcial esquerda: experiência do Hospital São Francisco. Rev Médica Sta Casa $P$. Alegre 1996; 8: 1506-13.

20 Lucchese F A, Frota Filho J D, Blacher C et al. - Partial left ventriculectomy: overall and late results in 44 class IV patients followed-up to 4 years. Ann Thorac Surg. [In press].

21 Lucchese F A - Partial left ventriculectomy according Batista procedure. Arch Chir Thorac Cardiovasc 1998; 20(1): jan-fev. (Editorial).

\section{Discussão (transcriçōes de fita gravada)}

\section{DR. JARBAS J. DINKHUYSEN}

São Paulo, SP

Que método foi utilizado para avaliar a resistência vascular pulmonar dos pacientes a cada três meses?

\section{DR. FROTA}

No caso destes pacientes que eu apresentei eles tiveram avaliações, a cada 6 meses, por cateterismo e não a cada 3 meses Os pacientes são avaliados por períodos de 6 meses até o final do primeiro ano e a partir disto, essas avaliações são passam a ser feitas anualmente.

\section{DR. JOÃO DE DEUS E BRITO \\ Rio de Janeiro , $R J$}

Parabéns pelo excelente trabalho, pois sei que você vem trabalhando neste assunto há muito tempo. Gostaria de fazer uma pergunta do ponto de vista técnico. Dr. Adib Jatene na correção geométrica do ventrículo esquerdo vem chamando atenção para o cuidado no tratamento do septo e nós sabemos que se você tem uma miocardiopadiatia dilatada e há um septo paradoxal eu acho que retirar apenas o músculo não vai adiantar, há necessidade de que o septo seja tratado como neste procedimento proposto pelo Randas. O Dor vem afirmando isto há muito tempo e o McCarthy, da Cleveland Clinic, também publicou um trabalho que excelentes resultados de uma operação modificada do Randas na miocardiopatia isquêmica dilatada. Nos casos que você operou houve necessidade de retirada de músculo e tratamento do septo? Na minha opinião não adianta tirar músculo se houver septo paradoxal.

\section{DR. FROTA \\ (Encerrando)}

O septo paradoxal, tanto quanto eu percebo, é muito freqüente na doença isquêmica. $\mathrm{Na}$ miocardiopatia dilatada idiopática é uma minoria de casos que pode apresentar movimento septal paradoxal. De fato, na miocardiopatia isquêmica se pretendemos atuar sobre o ventrículo também é necessário atuar sobre o septo, um dos componentes do ventrículo. $\mathrm{Na}$ miocardiopatia dilatada eu não vejo necessidade de atuar sobre o septo, desde que não seja isquêmica, seja idiopática. Exatamente aí reside um dos pitfalls, um dos segredos da ventriculectomia, ou seja, manter o septo tão íntegro, quanto possível, para que se mantenha sua reserva contrátil porque ele vai ser o elemento importante no novo ventrículo. De forma que, nos nossos pacientes como eram portadores de miocardiopatia dilatada, nós não intervimos no septo, em nenhum deles. Acredito que em pacientes com doença isquêmica, que têm fibrose septal ou que, por alguma razão, têm movimento septal paradoxal ou anômalo, s correção do septo é obrigatória. 